\title{
4 Die relative Chronologie der Früheisenzeit im südöstlichen Siebenstromland
}

Erste chronologische Studien für das Siebenstromland, vor allem für das südliche Siebenstromland, unternahm A. N. Bernštam in den I940er bis I950er Jahren. Anhand seiner Forschungen im südlichen Siebenstromland (sowohl im kasachischen als auch im kirgisischen Teil) erarbeitete er eine Kulturabfolge von der Bronzezeit bis zur jüngeren Eisenzeit. Der Schwerpunkt seiner Forschungen lag dabei aber überwiegend im südwestlichen Siebenstromland (nördliches Kirgisistan und Ču-Tal) und in der Alaj-Region, worauf er seine Überlegungen auch hauptsächlich stützte. Er entwickelte folgendes Chronologieschema (Bernštam i949b, Tab. I):

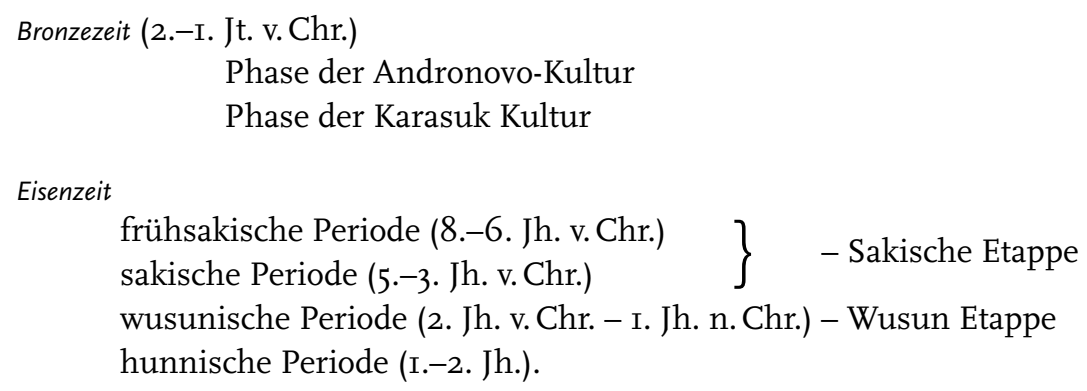

Da die Hinterlassenschaften der ersten drei eisenzeitlichen Perioden viele Ähnlichkeiten aufwiesen, verband Bernštam sie zur „Sako-Wusunischen Kultur“, die er in zwei Etappen untergliederte - die sakische und die wusunische Etappe (Bernštam I949b, 344) ${ }^{152}$.

Während der sakischen Etappe entstanden im Siebenstromland die Kurgangräberfelder. Die Kurgane bildeten eine oder mehreren Ketten, wobei die größten, die man als „Fürsten-Kurgane“ bezeichnen kann, stets in der Mitte standen. Die Kleinkurgane wurden am Rande des Gräberfeldes bzw. zwischen den Ketten der Großkurgane angelegt. Die Steinaufschüttung hatte im Grundriss eine runde oder ellipsoide Form. Zusätzlich wurde am Kurganrand noch ein Kromlech errichtet ${ }^{153}$ (Bernštam I949b, 344). Zum Fundrepertoire der sakischen Etappe gehörten unregelmäßig geformte unverzierte Keramik, die ohne Drehscheibe aus Tonwülsten oder -streifen aufgebaut worden war, und Bronzegegenstände, vor allem Waffen (siehe oben). Dolche, Messer und Pfeilspitzen aus sakischen Kurganen wiesen Parallelen zu Funden aus den Kurganen von Čimtjue und Ajri-tam im Oberen Talas-Gebiet auf, die in das 7.-5. Jh. v. Chr. datiert wurden (Bernštam I950a, I07).

Die Wusun-Etappe (in der Wende vom 3. zum 2. Jh. v. Chr. - I. Jh. n. Chr.) wird ebenso wie vorangehende Etappe hauptsächlich durch Gräberfelder und Grabfunde charakterisiert. Die Kurgane in den Nekropolen ähneln äußerlich den sakischen, doch es fehlen die Kromlechs, und die Kurganreihen liegen nun immer auf einer exakten Linie. Die Kurganenketten bestanden sowohl aus großen, die sich immer in der Mitte der Reihe befanden, als auch aus kleinen Kurganen ${ }^{154}$. Die Keramik aus den Gräbern

I52 Vgl. dazu oben ausführlicher im Abschnitt Forschungsgeschichte.

I53 Dieses Konstruktionselement eines großen Fürstenkurgans wurde im südöstlichen Siebenstromland nicht festgestellt.
I54 Dies sieht Bernštam als typisch für die Wusun-Etappe an. Die Kurgane sind jedoch auch auf den früheren, sakischen Gräberfeldern des südöstlichen Siebenstromlandes geläufig. 
der wusunischen Etappe ähnelte den sakischen Gefäßen (Töpfe, Kannen, Tassen, Schüsseln oder Pokale), die allerdings in einer anderen Technik - von textilen Modeln abgeformt - hergestellt worden war. Die Form der Pfeilspitzen änderte sich nicht, doch sie wurden nun aus Eisen bzw. Knochen hergestellt. Einen wesentlichen Unterschied zum Fundspektrum der sakischen Periode stellten die in wusunischer Zeit regelhaft anzutreffenden chinesischen Importe dar, wie Textilien, Lacke oder Nephrit (Bernštam I949b, 346; Bernštam i950a, I08).

Allerdings war es Bernštam trotz der beschriebenen Unterschiede nicht gelungen, die Charakterisierung der sakischen Denkmäler präzise zu fassen (Bernštam I949b, 346). Es gebe im nordkirgisischen Siebenstromland und in den Lösshügellandschaften des Ču-Tals extrem wenige frühsakische Fundplätze des 7.-6. Jh. v. Chr., so dass er sich überwiegend auf Zufalls- bzw. Lesefunde stützen musste (Bernštam I949b, 347). Dies erklärte er damit, dass die Träger der frühsakischen Sachkultur sich vor allem in den Hochgebirgstälern niedergelassen hätten und kaum die nördlich anschließenden Ebenen selbst bewohnten (Bernštam i950a, 106).

Aus der nachfolgenden Periode (3. Jh. v. Chr.-2. Jh. n. Chr.) waren bereits ausreichend wusunische Fundplätze im südlichen Siebenstromland bekannt, darunter auch einige wenige Siedlungsplätze. Allerdings war deren Zahl so gering, dass das Material aus den Gräbern nach wie vor die Grundlage bilden musste (Bernštam i950b, 59).

Kleine, unwesentliche Unterschiede in der Sachkultur der Saken und der Wusun könnten dadurch erklärt werden, dass die Wusun ein Teil des sakischen Kulturkreises waren. Die Wusun, auch als Issedonen bezeichnet, waren möglicherweise ein Stamm oder ein Stammesverband, der ab dem 3./2. Jh. v. Chr. bis zum I. Jh. n. Chr. den Machtbereich eines sakischen Stammes bzw. Stammesverbandes übernommen hatte. Ihre Wurzeln seien jedoch im selben Kulturkreis zu suchen, der allgemein als „sakisch“ bezeichnet wird (Bernštam I949b, 359). Bernštam folgerte aus chinesischen Schriftquellen, dass sich auf dem Gebiet der Wusun unterschiedliche Stämme niedergelassen hätten. Einige davon wären die Saken - „Sä“ in den chinesischen Quellen, die auch von antiken Autoren wie Strabo oder Herodot erwähnt wurden (Bernštam i950b, 60).

Im I. Jh. v. Chr. kamen die ersten hunnischen Verbände ins Siebenstromland (Bernštam I950b, 6o), was durch Katakombengräber und künstlich deformierte Schädel der Bestatteten belegt wird (Bernštam i949b, 359-365).

In einer Synthese neuer Grabungsergebnisse und historischer Informationen aus chinesischen Schriftquellen entwickelte A. N. Bernštam folgende ethnische Besiedlungsentwicklung im südlichen Siebenstromlandi55 (Bernštam I952, 27-72):

6.-5. Jh. v. Chr.: Sakische Verbände

5.-3. Jh. v. Chr.: Issedonische (Vorwusunische) Verbände

2. Jh. v. Chr. - 2. Jh. n. Chr.: Wusun-Yuezhi Verbände

I.-4. Jh. n. Chr.: Hunnische Verbände.

I96I gliederte E. I. Ageeva die Früheisenzeit in eine „sakische und wusunische Zeit“, indem sie die Wusun-Zeit anhand unterschiedlicher Keramikleitformen in drei Abschnitte unterteilte (Ageeva I96I, 65-66):

Bzw. des kirgisischen Tien-Shans. 
I) 3.-I. Jh. v. Chr.: Schüsseln und Krüge mit Rundböden;

2) I. Jh. v. Chr. - I. Jh. n. Chr.: bauchige Gefäße mit kleinen Standflächen und größere Variation

der Rand- und Halsprofile;

3) I.-3. Jh.: überwiegend flachbodige Keramik.

Nach zahlreichen Rettungsgrabungen im Ili-Tal in den i95oer und ig6oer Jahren analysierte G. A. Kušaev die Gräber der Wusun-Periode auf dem rechten, nördlichen Ili-Ufer, wobei er zu einer dreistufigen Untergliederung der Wusun-Kultur gelangte (Akišev/Kušaev I963, I48, I82, 208). Ihren chronologischen Rahmen sah er wie Ageeva vom 3. Jh. v. Chr. - 3. Jh. n. Chr.:

Wusun-Kultur: - Frühe Wusun-Etappe (3.-I. Jh. v Chr.)

- Mittlere Wusun-Etappe (I. Jh. v. Chr. - I. Jh. n. Chr.)

- Späte Wusun-Etappe (2.-3. Jh.).

In den Jahrzehnten zwischen I960 und I990 gab es hinsichtlich der Chronologie der älteren Eisenzeit bzw. der sakischen Periode im südöstlichen Siebenstromland keine entscheidenden Fortschritte. Erst im Jahre I992 legte Ju. A. Zadneprovskij eine neubearbeitete Periodisierung der Eisenzeit für das nördlichen Tien-Shan-Gebiet vor (Zadneprovskij I992, 75-87):

Frühsakische Periode: 8.-6. Jh. v. Chr.

Sakische Periode: 5.-3. Jh. v. Chr.

Wusun Periode: 3 . Jh. v. Chr. -5 . Jh.:

Frühwusun-Etappe: 3.-I. Jh. v. Chr.

Mittelwusun-Etappe: I. Jh. v. Chr. - 3. Jh. n. Chr.

Spätwusun-Etappe: 3.-5. Jh. n. Chr.

H. Parzinger (Parzinger 2006, Klapptafel III) gliederte die Eisenzeit in zwei Perioden (Tabelle 8): die ältere Eisenzeit (9./8.-3. Jh. v. Chr.) und die jüngere Eisenzeit (3. Jh. v. Chr. - Anfang des 5. Jh. n. Chr.). Für das Siebenstromland im nördlichen Tien-Shan nahm er Zadneprovskijs Ansätze auf (FrühsakischeStufe $\rightarrow$ Sakische-Stufe $\rightarrow$ Wusun-Stufe). In Anlehnung an die Gliederung in Südsibirien (Tuva: Aldy Bel'-Stufe; Minusinsker Becken: Podgornovo-Etappe) beschrieb er, vor allem für das mittlere bzw. zentrale Siebenstromland die frühsakische Biže-Stufe (7--6. Jh. v. Chr.). Weiterhin korrelierte er die Funde der darauffolgenden Issyk-Besšatyr-Stufe des südöstlichen Siebenstromlandes (5.-3. Jh. v. Chr.) mit den Funden aus Kurgan 5 vom Gräberfeld Čilikta in Ostkasachstan und aus dem Kurgan Aržan 2 in Tuva einerseits und der Keramik der Ejlatan-Kultur im Fergana-Tal andererseits (Parzinger 2006, 660-662). Beide Stufen, sowohl die Biže-Stufe als auch die Besšatyr-Stufe, gehörten seiner Meinung nach zur älteren Eisenzeit (Tabelle 8; vgl. Parzinger 2006, Klapptafel III). Ab dem 3. Jh. v. Chr. begann dann die jüngere Eisenzeit, die durch die Wusun-Periode mit ihren drei Stufen - frühe Wusun (3.-I. Jh. v. Chr./ I. Jh. n. Chr.) $\rightarrow$ mittlere Wusun (I. Jh. v. Chr./I. Jh. n. Chr. - 2. Jh.) $\rightarrow$ späte Wusun (2.-5. Jh.) - repräsentiert wurde (Tabelle 8; vgl. Parzinger 2006, 790-795, Klapptafel III).

Letztlich gelangte Parzinger zur Herausarbeitung von zwei wesentlichen chronologischen Horizonten der älteren Eisenzeit, einem frühsakisch/frühskythischen und einem sakisch/mittelskythischspätskythischen Horizont. Der erste, frühsakisch/frühskythische Horizont umfasst die Ejlatan-Kultur, die frühsakische Periode, die frühe Tasmola-Kultur, die Baitovo-Bekteniz-Stufe, die Čilikta-Stufe, die Tagar-Podgornovo-, Aldy Bel'- (Aržan 2) und Majemer-Stufe, Baitovo-Vorsargat-Stufe, die Bobrovskijund Vorob'evo-Stufe sowie die frühsauromatische und frühskythische Perioden und schließt damit den gesamten Raum vom Fergana-Tal, dem Siebenstromland und dem nördlichen Tien-Shan, über ganz 


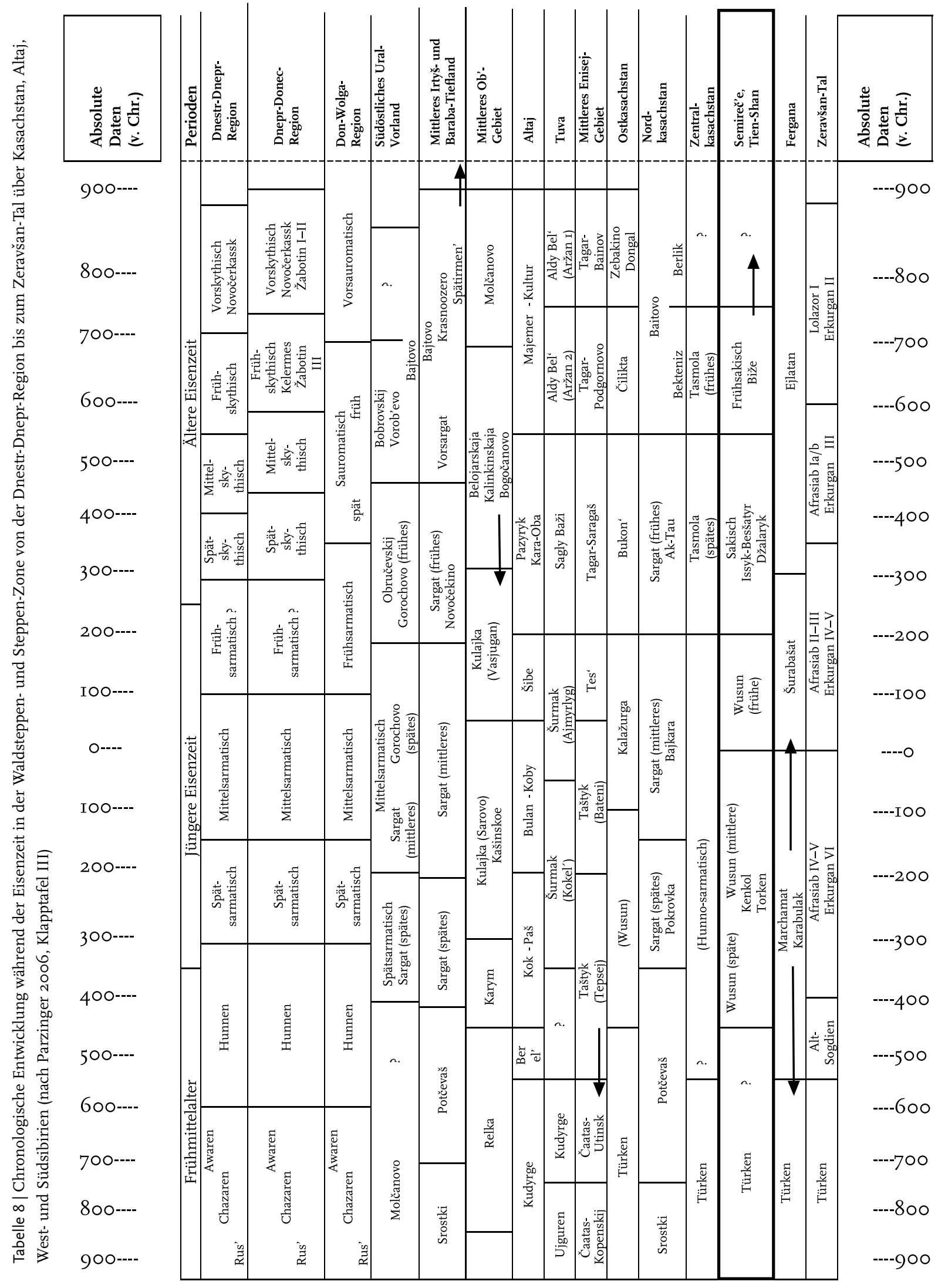


Kasachstan, Südsibirien, den Altaj und Westsibirien bis zum südöstlichen Ural-Vorland und weiter zur Don-Wolga-, Dnepr-Donec- und Dnestr-Dnepr Region (Parzinger 2006, Klapptafel III). Der sakisch/ mittelskythisch-spätskythische Horizont im gleichen Gebiet umfasst die Ejlatan-Kultur, die sakische Periode, die späte Stufe der Tasmola- und frühe Stufe der Sargat-Kultur, die Bukon-, Tagar-Saragaš- und Sagly Baži-Stufe, die Pazyryk-Kultur sowie spätsauromatische, frühsarmatische, mittel- und spätskythische Kulturen (Parzinger 2006, Klapptafel III).

\subsection{Zur absoluten Chronologie der Früheisenzeit}

Die chronologische Gliederung des südöstlichen Siebenstromlandes während der Eisenzeit bzw. Früheisenzeit kann mit Hilfe kalibrierter ${ }^{14} \mathrm{C}$-Daten abgesichert werden. Die ersten früheisenzeitlichen Datierungen wurden von der Kasachisch-Amerikanischen Expedition in der zweiten Hälfte der I99oer Jahre gewonnen. Es handelte sich um I3 Daten ${ }^{156}$ aus den Siedlungen Cyganka 8 und Tuzusaj im nördlichen Tien-Shan (Chang u.a. 2003, 303, Table I). Erst vor wenigen Jahren kam ein weiteres I4C-Datum dieser Epoche hinzu, und zwar aus einem früheisenzeitlichen Kurgan 3 aus Turgen $2^{157}$ (Gorjačev 2007 , I3). Demnach stehen zur absolutchronologischen Absicherung der Früheisenzeit im Siebenstromland lediglich vierzehn regionale ${ }^{\mathrm{I} 4 \mathrm{C}-D a t e n}$ zur Verfügung (Abb. 25).

Die veröffentlichten Radiokarbondaten weisen die datierten früheisenzeitlichen Objekte überwiegend in die zweite Hälfte des I. Jt. v. Chr. ${ }^{158}$. Diese Objekte wurden in die sakische Stufe eingeordnet (Zadneprovskij I992, 75-87; vgl. Parzinger 2006, 559-662, Klapptafel III).

Im Rahmen des Topoi-Teilprojektes „Archäologische und geoarchäologische Untersuchungen im südöstlichen Siebenstromland/ Kasachstan“ und der Ausgrabungen der Kasachisch-Deutschen Archäologischen Expedition wurden jüngst zehn weitere ${ }^{\mathrm{4}} \mathrm{C}$-Datierungen für die frühe Eisenzeit gewonnen 159 (vgl. Abb. 26). Dazu wurden in unterschiedlichen Labors verschiedene Materialien datiert: Menschen- und Tierknochen, Holz, verkohlte Makroreste sowie Rußpartikel aus Brandrückständen auf einem Opfertisch aus Bronze. Die Daten wurden aus den Nekropolen Žoan Tobe (Kurgan I, Objekt 2 sowie Kurgane 8 und 9) und Ulžan (Kurgan 2), aus der Peripherie des Kurgans 2 vom Plateau Kegen sowie aus den Kurganen des archäologischen Komplexes Turgen 2 und aus einem Hortfund in der Nähe des Gräberfeldes von Turgen gewonnen ${ }^{160}$.

Nach dem Zusammenfügen der neugewonnenen ${ }^{14} \mathrm{C}$-Daten mit den bereits veröffentlichten $\mathrm{Da}-$ ten ${ }^{16 I}$ (Tabelle 9) gewinnt man einen Überblick über die chronologische Entwicklung der Eisenzeit im Untersuchungsgebiet. Die neuen Daten haben die bereits bekannten im Wesentlichen bestätigt und

I56 $\mathrm{Zu}$ den Nachweisen im Einzelnen siehe Appendix I Nr. $25-37$.

I57 Vgl. zu diesem Datum Kap. I Forschungsgeschichte und Appendix I Nr. 24.

I58 Die beiden späten Daten (3./2. Jh. v. Chr.-I. Jh. n. Chr.) aus den Gruben I7 und 24 der Siedlung Tuzusaj (Tuzusaj 06 und Tuzusaj o7) gehören wahrscheinlich zur letzten Besiedlungsphase, die der Wusun-Stufe zugeordnet werden kann (Zadneprovskij I992, 75-87; vgl. Parzinger 2006, 790-795, Klapptafel III).

I59 Zwei datierte Befunde erwiesen sich als mittelalterlich. Die datierten Tierknochen aus Ulžan o2 (6.-7. Jh.) kön- nen als Überreste einer frühmittelalterlichen Trizna/Gedenkfeier interpretiert werden; bei der Bestattung von Žoan Tobe aus dem Io./II. Jh. handelt es sich um eine mittelalterliche Nachbestattung an der Peripherie eines sakischen Fürstenkurgans (Abb. 26; Tabelle 9).

I60 Vgl. zu näheren Informationen die Einträge im Katalog. Z. Samašev, K. Altynbekov, M. Nurpeisov und A. Gorjačev danke ich für die Bereitstellung von Probenmaterial zur Datierung, H. Parzinger, A. Nagler, M. Nawroth und M.-R. Boroffka für die zur Verfügung gestellten unveröffentlichten Daten.

I6I Siehe auch Appendix I, Liste I und 2. 


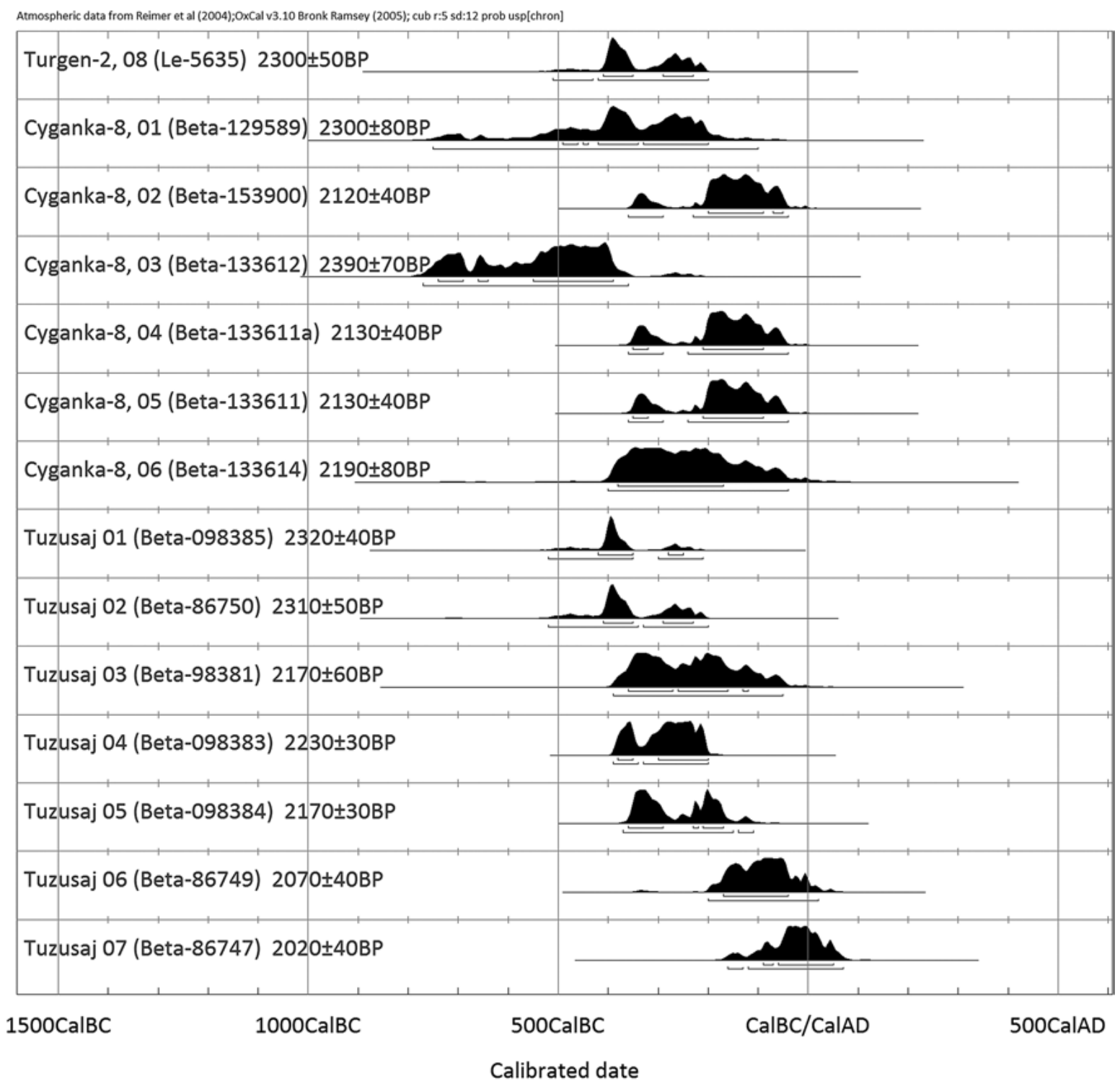

Abb. 25| Die Radiokarbondaten aus dem südöstlichen Siebenstromland (archäologischer Komplex Turgen 2, Siedlungen Cyganka 8 und Tuzusaj)

untermauern damit die Datierung der Hauptaktivitäten des Menschen während der Eisenzeit im nördlichen Tien-Shan in die zweite Hälfte des I. Jt. v. Chr. (6./5.-3./2. Jh. v. Chr.). Es handelt sich hierbei um die früh- bzw. ältereisenzeitliche sakische Stufe (Zadneprovskij I992, 75-87; Parzinger 2006, 559-662, Klapptafel III).

Die frühsakische Zeitstufe (7.-6. Jh. v. Chr.) konnte bislang im Untersuchungsgebiet nicht sicher gefasst werden, das Gleiche gilt auch für die Übergangszeit von der Spätbronze- zur Früheisenzeit (9.-8. Jh. v. Chr.). Es gibt allerdings einige Indizien, die für eine Besiedlung des südöstlichen Siebenstromlandes in diesen Zeiträumen sprechen könnten. Eine separate Pferdebestattung in Kurgan I2 des Gräberfeldes Džuvantobe wurde aufgrund der Trensen mit Steigbügelenden und der Sattelgurtschnal- 


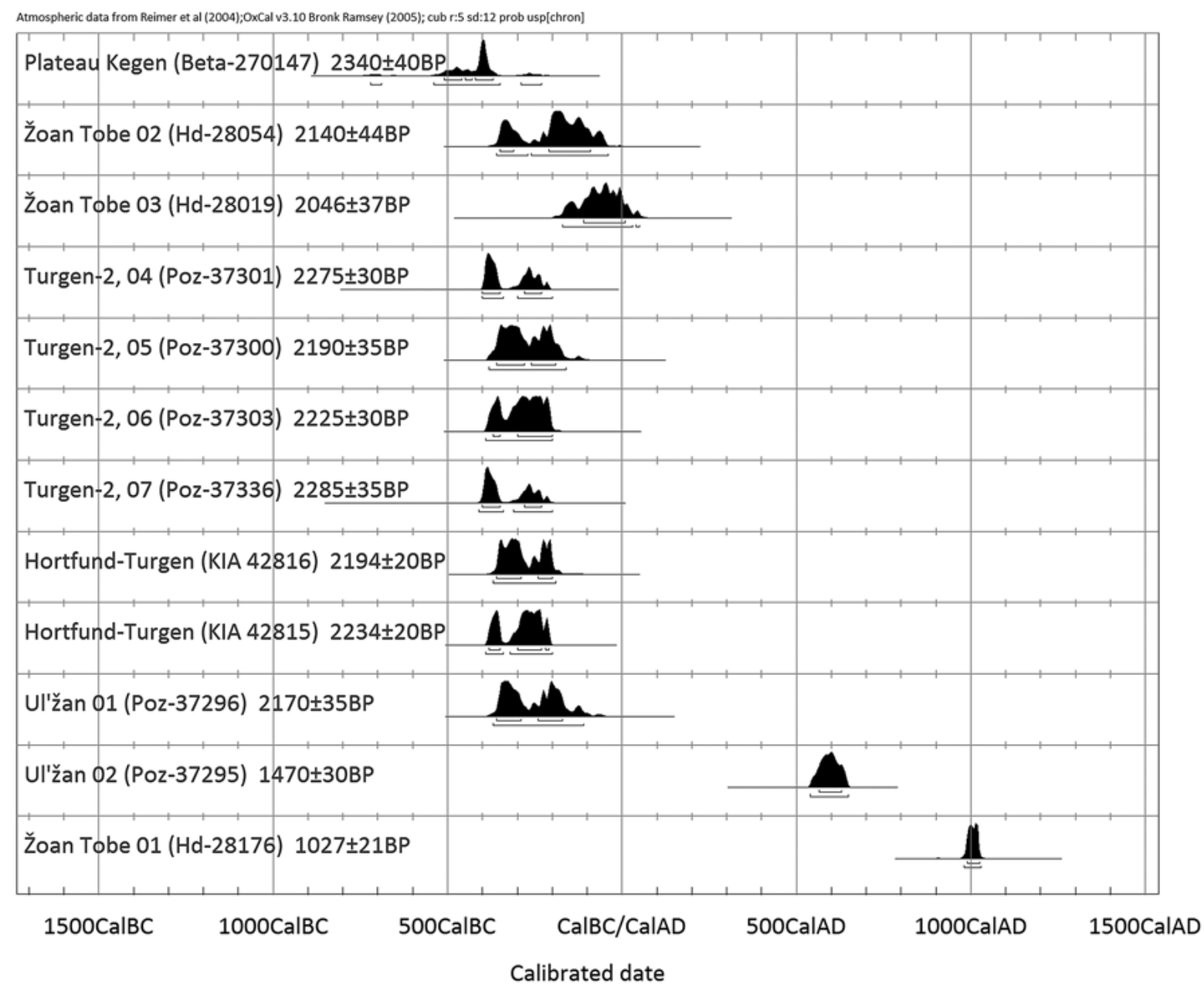

Abb. 26 | Die neugewonnenen Radiokarbondaten aus dem südöstlichen Siebenstromland ${ }^{162}$

len ${ }^{163}$ in das 7.-6. Jh. v. Chr. datiert (Maksimova I96oa, 62, 64; vgl. auch Katalog). Grund dafür waren Parallelen aus den Majemer-Kurganen im Altaj, aus Gräbern im mittleren Irtyš- und Dnepr-Gebiet, in der Tjup-Gegend (Kirgisistan) und in der Taškent-Oase. Auch einige Gegenstände aus dem „Schatzfund vom Žalauly “' ${ }^{6} 4$ konnten aufgrund von Analogien mit Gegenständen aus dem Kurgan I von Čilikty-2 in das 7. Jh. v. Chr. datiert werden (Samašev 2007, I64-165; Samašev u.a. 2007, I6-I8, 24-3I; vgl. auch Katalog, Ergänzung zu zwei Kurganen $400 \mathrm{~m}$ westlich vom Dorf Žalauly).

Diese Indizien können keinesfalls eine Besiedlung des südöstlichen Siebenstromlandes in der Übergangszeit von der Bronze- zur Eisenzeit und in der frühsakischen Zeitstufe belegen. Nach dem gegenwärtigen Kenntnisstand muss demnach davon ausgegangen werden, dass das Untersuchungsgebiet in dieser Periode keine nennenswerte Besiedlung aufwies.

I62 Nachweise zu den Proben im Einzelnen: Appendix I Nr. 9-20.

I63 Dieser Befund und das Pferdegeschirr sind bis heute im südöstlichen Siebenstromland einmalig.
I64 Wie z. B. ein goldener Beschlag mit zwei antithetisch angeordneten Hirschen, deren Geweihe zusammenlaufen. 
Tabelle 9 | Gesamtübersicht über alle verfügbaren ${ }^{14}$ C-Daten zur Früheisenzeit im südöstlichen Siebenstromland

\begin{tabular}{|c|c|c|c|c|c|}
\hline Probe & $\begin{array}{l}\text { Labor- } \\
\text { nummer }\end{array}$ & Fundort & Material & $\begin{array}{l}\text { Radio- } \\
\text { karbonalter }\end{array}$ & $\begin{array}{l}\text { Kalibriertes } \\
\text { Datum } \\
95,4 \% \text { probability } \\
\end{array}$ \\
\hline Plateau Kegen & Beta-270I47 & $\begin{array}{l}\text { Gf. auf dem Plateau Kegen, } \\
\text { Kurgan 2, nördlicher } \\
\text { Kurganrand, Fläche I }\end{array}$ & $\begin{array}{l}\text { Tierkno- } \\
\text { chen }\end{array}$ & $2340 \pm 40 \mathrm{BP}$ & $\begin{array}{l}720-690 \mathrm{cal} \mathrm{BC} \mathrm{(I,4 \% )} \\
540-350 \mathrm{cal} \mathrm{BC} \\
(89,5 \%) \\
290-230 \mathrm{cal} \mathrm{BC} \\
(4,5 \%)\end{array}$ \\
\hline Žoan Tobe 02 & Hd-28054 & Gf. Žoan Tobe, Kurgan 8 & Holz & $2 \mathrm{I} 40 \pm 44 \mathrm{BP}$ & $\begin{array}{l}360-270 \text { cal BC } \\
(23,5 \%) \\
260-40 \text { cal BC } \\
(71,9 \%)\end{array}$ \\
\hline Žoan Tobe 03 & Hd-28oI9 & Gf. Žoan Tobe, Kurgan 9 & $\begin{array}{l}\text { botanisches } \\
\text { Material }\end{array}$ & $2046 \pm 37 \mathrm{BP}$ & 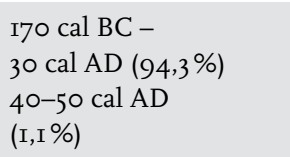 \\
\hline Turgen-2, ०4 & Poz-3730I & $\begin{array}{l}\text { Kpx. Turgen-2, Kurgan 7, } \\
\text { Grab I, Bestattung I }\end{array}$ & $\begin{array}{l}\text { Knochen } \\
\text { (Homo) }\end{array}$ & $2275 \pm 30 \mathrm{BP}$ & $\begin{array}{l}400-340 \text { cal BC } \\
(5 \mathrm{I}, \mathrm{I} \%) \\
300-200 \mathrm{cal} \mathrm{BC} \\
(44,3 \%)\end{array}$ \\
\hline Turgen-2, 05 & Poz-37300 & $\begin{array}{l}\text { Kpx. Turgen-2, Kurgan 7, } \\
\text { Grab I, Bestattung } 2\end{array}$ & Holz & $2190 \pm 35 \mathrm{BP}$ & $\begin{array}{l}380-\mathrm{I} 60 \mathrm{cal} \text { BC } \\
(95,4 \%)\end{array}$ \\
\hline Turgen-2, ०6 & Poz-37303 & $\begin{array}{l}\text { Kpx. Turgen-2, Kurgan Io, } \\
\text { Grab I, Bestattung I }\end{array}$ & $\begin{array}{l}\text { Knochen } \\
\text { (Homo) }\end{array}$ & $2225 \pm 30 \mathrm{BP}$ & $\begin{array}{l}390-200 \mathrm{cal} \mathrm{BC} \\
(95,4 \%)\end{array}$ \\
\hline Turgen-2, 07 & Poz-37336 & $\begin{array}{l}\text { Kpx. Turgen-2, Kurgan Io, } \\
\text { Grab I, Bestattung } 2\end{array}$ & $\begin{array}{l}\text { Knochen } \\
\text { (Homo) }\end{array}$ & $2285 \pm 35 \mathrm{BP}$ & $\begin{array}{l}4 \mathrm{IO}-340 \mathrm{cal} \mathrm{BC} \\
(54,0 \%) \\
3 \mathrm{IO}-200 \mathrm{cal} \mathrm{BC} \\
(4 \mathrm{I}, 4 \%)\end{array}$ \\
\hline Hortfund-Turgen & KIA $428 \mathrm{I} 6$ & $\begin{array}{l}\text { Hortfund beim Gräberfeld } \\
\text { Turgen, Opfertisch, } \\
\text { Probe } 23 \\
\text { (N43 25.I37 } \\
\text { E77 34.6I3) }\end{array}$ & Ruß & $2194 \pm 20 \mathrm{BP}$ & $\begin{array}{l}370-190 \mathrm{cal} \mathrm{BC} \\
(95,4 \%)\end{array}$ \\
\hline Hortfund-Turgen & KIA $428 \mathrm{I} 5$ & $\begin{array}{l}\text { Hortfund beim Gräberfeld } \\
\text { Turgen, Opfertisch, } \\
\text { Probe } 5\end{array}$ & Ruß & $2234 \pm 20 \mathrm{BP}$ & $\begin{array}{l}390-340 \mathrm{cal} \mathrm{BC}(23, \mathrm{I} \%) \\
320-200 \mathrm{cal} \mathrm{BC} \\
(72,3 \%)\end{array}$ \\
\hline Ulžan or & Poz-37296 & Gf. Ulžan, Kurgan 2 & Holz & $2170 \pm 35 \mathrm{BP}$ & 370-IIo cal BC $(95,4 \%)$ \\
\hline Turgen-2, $08^{165}$ & Le- 5635 & Kpx. Turgen-2, Kurgan 3 & Holz & $2300 \pm 50 \mathrm{BP}$ & $\begin{array}{l}5 \mathrm{IO}-430 \mathrm{cal} \mathrm{BC}(5, \mathrm{I} \%) \\
420-200 \mathrm{cal} \mathrm{BC} \\
(90,3 \%)\end{array}$ \\
\hline Cyganka-8, oI ${ }^{166}$ & $\begin{array}{l}\text { Be- } \\
\text { ta-I29589 }\end{array}$ & $\begin{array}{l}\text { Sdl. Cyganka } 8 \text {, Schicht I, } \\
\text { Vorratsgrube } 98\end{array}$ & & $2300 \pm 80 \mathrm{BP}$ & 750-I00 cal BC (95,4\%) \\
\hline Cyganka-8, o2 & Beta-I53900 & $\begin{array}{l}\text { Sdl. Cyganka 8, Schicht II, } \\
\text { Haus 3, Bodenhorizont } 4\end{array}$ & & $2 \mathrm{I} 20 \pm 40 \mathrm{BP}$ & $\begin{array}{l}360-290 \text { cal BC } \\
(\text { (10,9\%) } \\
230-40 \text { cal BC } \\
(84,5 \%)\end{array}$ \\
\hline Cyganka-8, ०3 & Beta-1336I2 & $\begin{array}{l}\text { Sdl. Cyganka 8, Schicht III, } \\
\text { Haus 3, Bodenhorizont } \\
\text { 3a/b }\end{array}$ & & $2390 \pm 70 \mathrm{BP}$ & $770-360 \mathrm{cal} \mathrm{BC}(95,4 \%)$ \\
\hline
\end{tabular}

I65 Die Angaben und das Radiokarbonalter nach Gorjačev 2007b, I3.
I66 Alle Angaben von Cyganka 8 und Tuzusaj nach Chang u.a. 2003, 303, Table I. 


\begin{tabular}{|c|c|c|c|c|c|}
\hline Probe & $\begin{array}{l}\text { Labor- } \\
\text { nummer }\end{array}$ & Fundort & Material & $\begin{array}{l}\text { Radio- } \\
\text { karbonalter }\end{array}$ & $\begin{array}{l}\text { Kalibriertes } \\
\text { Datum } \\
95,4 \% \text { probability }\end{array}$ \\
\hline Cyganka-8, ०4 & $\begin{array}{l}\text { Be- } \\
\text { ta-I336ria }\end{array}$ & $\begin{array}{l}\text { Sdl. Cyganka 8, Haus 2, } \\
\text { Bodenhorizont } 2\end{array}$ & & $2 \mathrm{I} 30 \pm 40 \mathrm{BP}$ & $\begin{array}{l}360-290 \mathrm{cal} \mathrm{BC}(\mathrm{I} 6, \mathrm{I} \%) \\
240-40 \mathrm{cal} \mathrm{BC} \\
(79,3 \%)\end{array}$ \\
\hline Cyganka-8, ०5 & Beta-I336II & Sdl. Cyganka 8, Grube I3 & & $2 \mathrm{I} 30 \pm 40 \mathrm{BP}$ & $\begin{array}{l}360-290 \mathrm{cal} \mathrm{BC}(\mathrm{I} 6, \mathrm{I} \%) \\
240-40 \mathrm{cal} \mathrm{BC} \\
(79,3 \%)\end{array}$ \\
\hline Cyganka-8, o6 & Beta-I336I4 & $\begin{array}{l}\text { Sdl. Cyganka 8, Schicht } \\
\text { VIa, Anlage V-Io }\end{array}$ & & $2190 \pm 80 \mathrm{BP}$ & $400-40 \mathrm{cal} \mathrm{BC}(95,4 \%)$ \\
\hline Tuzusaj о I & $\begin{array}{l}\text { Be- } \\
\text { ta-og } 8385\end{array}$ & Sdl. Tuzusaj, Grube 8 & & $2320 \pm 40 \mathrm{BP}$ & $\begin{array}{l}520-350 \mathrm{cal} \mathrm{BC}(78,3 \%) \\
300-2 \mathrm{IO} \text { cal BC } \\
(\mathrm{I} 7, \mathrm{I} \%)\end{array}$ \\
\hline Tuzusaj 02 & Beta- 86750 & $\begin{array}{l}\text { Sdl. Tuzusaj, Schicht IV, } \\
\text { Grube } 22\end{array}$ & & $23 \mathrm{IO} \pm 50 \mathrm{BP}$ & $\begin{array}{l}520-340 \mathrm{cal} \mathrm{BC}(6 \mathrm{I}, 6 \%) \\
330-200 \mathrm{cal} \mathrm{BC} \\
(33,8 \%)\end{array}$ \\
\hline Tuzusaj 03 & Beta-9838I & $\begin{array}{l}\text { Sdl. Tuzusaj, Anlage V-I3, } \\
\text { Ascheablagerung }\end{array}$ & & $2 \mathrm{I} 70 \pm 60 \mathrm{BP}$ & $390-50 \mathrm{cal} \mathrm{BC}(95,4 \%)$ \\
\hline Tuzusaj ०4 & $\begin{array}{l}\text { Be- } \\
\text { ta-og } 8383\end{array}$ & Sdl. Tuzusaj, Grube 29 & & $2230 \pm 30 \mathrm{BP}$ & $\begin{array}{l}390-340 \mathrm{cal} \mathrm{BC}(23,0 \%) \\
330-200 \mathrm{cal} \mathrm{BC} \\
(72,4 \%)\end{array}$ \\
\hline Tuzusaj 05 & $\begin{array}{l}\text { Be- } \\
\text { ta-og } 8384\end{array}$ & $\begin{array}{l}\text { Sdl. Tuzusaj, Schicht V, } \\
\text { Grube } 30 \text { B }\end{array}$ & & $2170 \pm 30 \mathrm{BP}$ & $\begin{array}{l}370-I 50 \mathrm{cal} \mathrm{BC}(92,6 \%) \\
\text { I40-IIO cal BC } \\
(2,8 \%)\end{array}$ \\
\hline Tuzusaj o6 & Beta- 86749 & $\begin{array}{l}\text { Sdl. Tuzusaj, Schicht VI, } \\
\text { Grube I7 }\end{array}$ & & $2070 \pm 40 \mathrm{BP}$ & $\begin{array}{l}200 \mathrm{cal} \mathrm{BC}- \\
20 \mathrm{cal} \mathrm{AD}(95,4 \%)\end{array}$ \\
\hline Tuzusaj o7 & Beta- 86747 & $\begin{array}{l}\text { Sdl. Tuzusaj, Schicht VI, } \\
\text { Grube } 24\end{array}$ & & $2020 \pm 40 \mathrm{BP}$ & $\begin{array}{l}\text { I60-I30 cal BC (4,3\%) } \\
\text { I20 cal BC - } \\
70 \mathrm{cal} \mathrm{AD} \mathrm{(9I,I \% )}\end{array}$ \\
\hline
\end{tabular}

In Tabelle ıo ist das Chronologieschema für das südöstliche Siebenstromland wiedergegeben, das auf der Grundlage der chronologischen Übersicht von H. Parzinger (Parzinger 2006, Klapptafel III) erarbeitet wurde, indem es mit den neuen ${ }^{14} \mathrm{C}$-Datierungen aktualisiert wurde ${ }^{\mathrm{I} 67}$.

Abschließend ergibt sich aus der Zusammenfassung aller Informationen das folgende Chronologieschema der kulturellen Entwicklung im südöstlichen Siebenstromland (Tabelle II).

Diese schematische Darstellung der chronologischen Entwicklung im Untersuchungsgebiet kann selbstverständlich nicht als endgültig angesehen werden. Es stellt jedoch den gegenwärtigen Forschungsstand zur Bronze- und Eisenzeit im nördlichen Tien-Shan dar. Für eine Verbesserung und Erweiterung unserer Kenntnisse sind weitere intensive Ausgrabungen, Analysen und Datierungen unbedingt notwendig.

I67 Die von H. Parzinger erarbeitete frühsakische BižeStufe bezieht sich auf das mittlere bzw. zentrale Sieben- stromland (Parzinger 2006, 659), im südöstlichen Siebenstromland gibt es sie nicht. 
Tabelle 10 | Schematische Darstellung der chronologischen Entwicklung der Eisenzeit im südöstlichen Siebenstromland (z.T. nach Parzinger 2006, Klapptafel III)

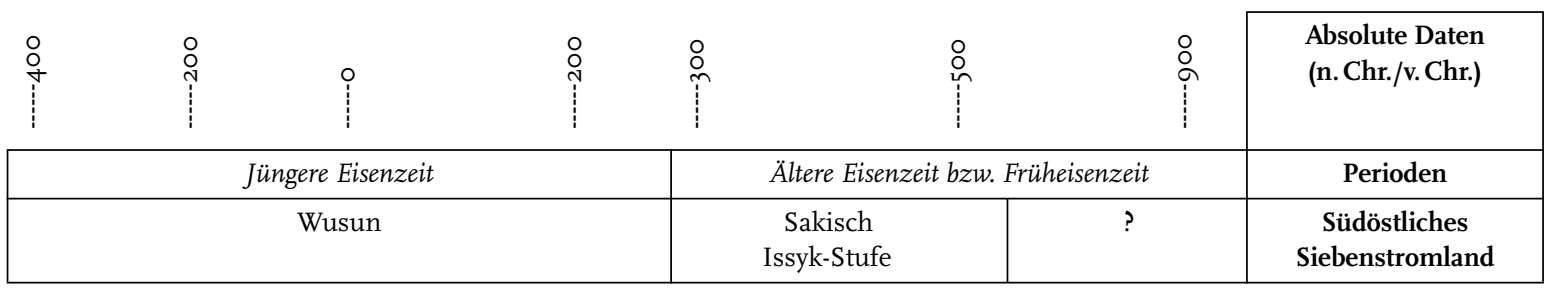

Tabelle 11 | Schematische Darstellung der chronologischen Entwicklung des südöstlichen Siebenstromlandes während der Bronze- und Eisenzeit

\begin{tabular}{|l|l|l|}
\hline Periode & Absolute Daten & Kultur/ Gruppe/ Stufe \\
\hline Frühbronzezeit & 25.-19. Jh. v. Chr. & ? \\
\hline Mittelbronzezeit & I9./I8.-I4. Jh. v. Chr. & $\begin{array}{l}\text { Andronovo-Fedorovka-Kultur } \\
\text { Kul'saj-Gruppe }\end{array}$ \\
\hline \multirow{2}{*}{ Spätbronzezeit } & I4./I3.-IO. Jh. v. Chr. & $\begin{array}{l}\text { Andronovo-Kultur } \\
\text { Etappe der Wulstkeramik ? - } \\
\text { nicht ausreichend belegt }\end{array}$ \\
\hline $\begin{array}{l}\text { Übergangszeit von Spätbronze- zur } \\
\text { Früheisenzeit (älteren Eisenzeit) }\end{array}$ & 9.-8 Jh. v. Chr. & ? \\
\hline \multirow{2}{*}{ Ältere Eisenzeit bzw. Früheisenzeit } & 8./7.-6. Jh. v. Chr. & Frühsakische Stufe ? - fehlt \\
\cline { 2 - 3 } & 6./5.-3./2. Jh. v. Chr. & Sakische-Stufe \\
\hline Jüngere Eisenzeit & 3./2. Jh. v. Chr.-5. Jh. n. Chr. & Wusun \\
\hline
\end{tabular}

